\title{
Impact of tozinameran (BNT162b2) mRNA vaccine on kidney transplant and chronic dialysis patients: 3-5 months follow-up
}

\author{
Iddo Z. Ben-Dov ${ }^{1}$ (D) Y Yonatan Oster ${ }^{2} \cdot$ Keren Tzukert $^{1} \cdot$ Talia Alster $^{1} \cdot$ Raneem Bader $^{3} \cdot$ Ruth Israeli $^{1} \cdot$ Haya Asayag $^{1}$. \\ Michal Aharon ${ }^{1} \cdot$ Ido Burstein $^{1} \cdot$ Hadas Pri-Chen ${ }^{1}$. Ashraf Imam ${ }^{3}$ - Roy Abel ${ }^{1}$. Irit Mor-Yosef Levi ${ }^{1}$ - Abed Khalaileh ${ }^{3}$. \\ Esther Oiknine-Djian ${ }^{2} \cdot$ Aharon Bloch $^{1} \cdot$ Dana G. Wolf ${ }^{2} \cdot$ Michal Dranitzki Elhalel $^{1}$
}

Received: 1 August 2021 / Accepted: 11 November 2021 / Published online: 6 January 2022

(c) The Author(s) under exclusive licence to Italian Society of Nephrology 2021

\begin{abstract}
Background Determining the humoral immunogenicity of tozinameran (BNT162b2) in patients requiring chronic renal replacement therapy, and its impact on COVID-19 morbidity several months after vaccination, may guide risk assessment and changes in vaccination policy.

Methods In a prospective post-vaccination cohort study with up to 5 months follow-up we studied outpatient dialysis and kidney transplant patients and respective healthcare teams. Outcomes were anti S1/S2 antibody responses to vaccine or infection, and infection rate during follow-up.

Results One hundred seventy-five dialysis patients (40\% women, $65 \pm 15$ years), 252 kidney transplant patients ( $33 \%$ women, $54 \pm 14$ years) and 71 controls ( $65 \%$ women, $44 \pm 14$ years) were followed. Three months or longer after vaccination we detected anti $\mathrm{S} 1 / \mathrm{S} 2 \mathrm{IgG}$ antibodies in $79 \%$ of dialysis patients, $42 \%$ of transplant recipients and $100 \%$ of controls, whereas respective rates after infection were $94 \%, 69 \%$ and $100 \%$. Predictors of non-response were older age, diabetes, history of cancer, lower lymphocyte count and lower vitamin-D levels. Factors associated with lower antibody levels in dialysis patients were modality (hemodialysis vs peritoneal) and high serum ferritin levels. In transplant patients, hypertension and higher calcineurin or mTOR inhibitor drug levels were linked with lower antibody response. Vaccination was associated with fewer subsequent infections (HR 0.23, p < 0.05). Moreover, higher antibody levels (particularly above $59 \mathrm{AU} / \mathrm{ml}$ ) were associated with fewer events, with a HR 0.41 for each unit increased in $\log _{10}$ titer $(\mathrm{p}<0.05)$.

Conclusions Dialysis patients, and more strikingly kidney transplant recipients, mounted reduced antibody response to COVID-19 mRNA vaccination. Lesser humoral response was associated with more infections. Measures to identify and protect non-responsive patients are required.
\end{abstract}

\section{Graphic abstract}

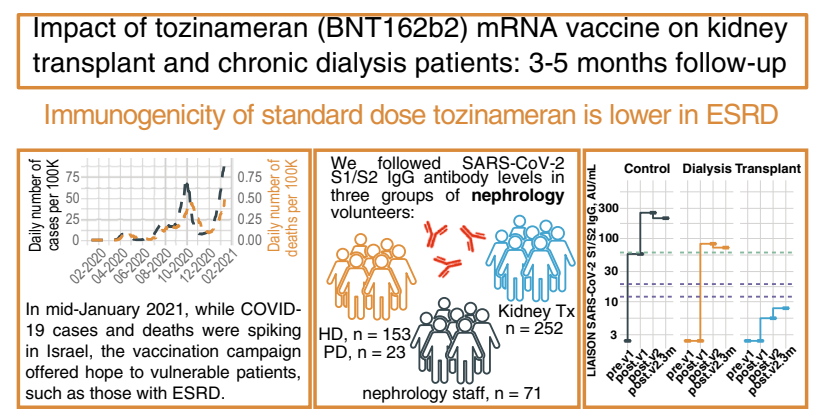

Keywords COVID-19 Viral infections · Vaccines · End-stage renal disease $\cdot$ Kidney transplantation $\cdot$ Hemodialysis . Peritoneal dialysis

Extended author information available on the last page of the article 


\section{Introduction}

Vaccinations, both passive and active, have changed the natural course of many infectious diseases, affecting both spread and severity. From smallpox through rabies and measles, to hepatitis B, as well as many other viral and bacterial infections, vaccines have either eradicated diseases or eliminated the risk for pandemic or endemic catastrophes [1,2]. Therefore, efforts to develop an effective vaccination against COVID-19 started as soon as the impact of this coronavirus on global public health was appreciated. Impressively, effective vaccines were developed and approved in record time, within less than a year. These active vaccines offer hope for controlling disease spread and reducing illness severity and death rates. Various vaccines are already in use, and data emerging from real-world practice (as from Israel [3] and other countries) suggest effectiveness in the prevention of severe disease [4].

The COVID-19 pandemic brought new challenges to the general population and even more so to patients with endstage renal disease (ESRD), many of whom have comorbidities now acknowledged as risk factors for severe COVID-19 [5]. Aside from the logistic challenges summarized elsewhere [6], these patients, both dialysis-treated and kidney transplant patients, face morbidity and mortality risks that are significantly higher than the general population $[7,8]$. Unfortunately, it is known from existing vaccine preparations such as anti-hepatitis B virus and influenza [9-12], that both dialysis and kidney transplant patients require higher doses and repeat inoculations (i.e. periodic boosts) in order to achieve durable protection [13, 14]. Uremia, age, comorbidities such as diabetes, and nutritional status have been shown to associate with reduced immune response to HBV vaccines. This diminished response to vaccination is thought to be secondary to dysfunction of both B and $\mathrm{T}$ lymphocytes in ESRD patients, as well as to the immunosuppressant medications taken by transplant recipients (occasionally also after resuming dialysis). Dendritic cell dysfunction, described in patients with ESRD [15] likely compromises vaccination success. In the general population, a robust correlation was seen between anti-SARS-CoV-2 neutralizing titer and efficacy and binding antibody titer and efficacy [16]. However, information available about COVID19 vaccine effectiveness in dialysis and transplanted patients is limited and short-termed [17, 18], and thus we sought to prospectively follow antibody development in these patients, to compare them with healthy controls and to identify risk factors for diminished humoral response and disease.

\section{Methods}

This prospective study was designed to follow IgG antibody levels after COVID-19 vaccination and/or infection in kidney transplant patients and patients requiring maintenance dialysis, and to estimate associations between vaccine inoculation, the resulting humoral response and subsequent COVID-19 infections. Nephrology healthcare personnel served as controls.

\section{Participants and setting}

Immunization with tozinameran, Pfizer and BioNTech's BNT162b2 vaccine, commenced in Israel on 12-Dec2020, and immunocompromised patients were among the first priority groups. Prior to initiation of vaccination we launched the COVID-19 mRNA Vaccine Immunogenicity in patients with end-stage Renal Disease (COVIReD) prospective cohort study designed to investigate the long-term kinetics and implications of antibody response to COVID19 vaccine and infection in this vulnerable population. We are characterizing the humoral response to COVID-19 infection and vaccination as well as disease occurrence among patients treated with maintenance dialysis, kidney transplant recipients (regardless of allograft function) and control subjects at Hadassah Medical Center, a two-campus tertiary medical center in Jerusalem, Israel. Jerusalem was the area with the highest COVID-19 prevalence in Israel $[19,20]$ and since the beginning of the pandemic, more than 4,500 COVID-19 patients have been admitted to our institution. We recruited control subjects from amongst medical, nursing and administrative healthcare staff at the dialysis unit and/or the transplantation clinic.

\section{Clinical methods}

All procedures performed in this study were in accordance with the ethical standards of the institutional Helsinki Committee and with the 1964 Helsinki Declaration and its later amendments. Written informed consent was obtained from participants.

The study protocol included documentation of vaccination, but not the act of vaccination or monitoring for adverse effects. Adults in Israel that had not been previously infected with COVID-19 were offered, beginning in late December 2020, two tozinameran (BNT162b2 vaccine) injections, at the manufacturer's standard recommended dose, 3 weeks apart. The 2 nd dose was omitted in subjects infected with COVID-19 within the interval between the 1st and intended 2nd dose. Subjects who have had COVID-19 before vaccination were offered a single 
vaccine dose ( $\geq 5$ months after infection). Blood samples were given by dialysis and transplant patients during routine visits, at several designated time-windows during the vaccination period: at baseline (namely, before administration of the first vaccine dose), 10-20 days after the first vaccine, 2-6 weeks after the second vaccine inoculation (typically scheduled 21 days after the first inoculation) and 3 months or longer after the first vaccination. The precise timing of blood sampling was not uniform between or within the study groups because there were no dedicated study visits, but rather blood was withdrawn at the time of blood sampling for routine clinical indications. Most hemodialysis patients and controls provided samples at all or most time-points, while transplant and peritoneal dialysis patients provided samples sporadically during their scheduled outpatient visits. We followed up participants for overall and COVID-free survival until 31-May-2021.

\section{Laboratory methods}

All serum samples were tested at Hadassah's clinical virology laboratory, using kits supplied by the Israeli Ministry of Health. Anti-SARS-2 IgG antibodies were quantified using LIAISON SARS-CoV-2 S1/S2 IgG (DiaSorin) and ARCHITECT SARS-CoV-2 IgG II (Abbott) immunoassays, and the former kit results are reported here. According to the recommendation of the State of Israel's Ministry of Health, results were categorized as negative when $<12$ $\mathrm{AU} / \mathrm{ml}$, borderline (equivocal) when $\geq 12$ but $<19 \mathrm{AU} / \mathrm{ml}$ and positive when $\geq 19 \mathrm{AU} / \mathrm{ml}$. In addition, before as well as after vaccination, patients were tested for viral RNA using qPCR assays when clinically or epidemiologically indicated, but we did not perform routine PCR screening except for staff. Inpatient and outpatient diagnoses, patient demographics and serology, virology and additional selected laboratory results (see Table 1) were extracted by the institution's information system for the period between 01-Mar-2020 and patient vaccination.

\section{Statistical analysis}

Deidentified clinical information and laboratory data and metadata were stored as spreadsheets and processed using $\mathrm{R}$ (R Core Team, 2021) [21] and related statistical packages. Clinical characteristics of study participants were summarized by group using means \pm SD or medians and ranges, as appropriate. Right-skewed variables (e.g. antibody levels) were $\log _{10}$-transformed prior to statistical testing. Betweengroup differences in baseline clinical characteristics were assessed using ANOVA or chi squared test. Statistical symbols embedded within plots reflect Mann-Whitney $U$ test results. Potential clinical predictors of antibody response and disease (COVID-19 infection) were examined using mixed-effects linear for numeric antibody levels, generalized linear models for dichotomous antibody results and Cox proportional hazards models, respectively, using 'lme4' [22], 'coxme' (mixed effects cox models) and 'survival' [23] packages. In models of antibody response, measurements performed in patients after COVID-19 infection were excluded, thus only response to vaccination is modeled and predicted. The tested independent predictor variables were renal replacement status (dialysis, transplant or control), age, sex, number of vaccine inoculations received, comorbidities (chronic obstructive pulmonary disease, obesity, hypothyroidism, cancer, cirrhosis, anemia, smoking, hyperlipidemia, hypertension, peripheral vascular disease and diabetes mellitus) and laboratory results (C-reactive protein, ferritin, hemoglobin, white blood cell count, lymphocyte count, albumin and vitamin D). In addition, in models restricted to patients receiving dialysis we included the dialysis vintage and 3-months' averaged urea reduction rate (URR, a surrogate of dialysis adequacy), while in models restricted to transplant patients we included time since transplantation, donor type (living or cadaver), estimated glomerular filtration rate (eGFR) and calcineurin or mTOR inhibitor trough levels. Time post-vaccination was modeled as an independent variable using splines, and the time $\times$ group interaction was also included in some models. Missing data were not imputed. We accounted for repeated measurements (from the same subject) by including a participant identifier as a random effect in these models. Model outputs are presented using the 'sjPlot' package [24]. Antibody level or positivity rate predictions based on these models were generated and plotted using the 'ggeffects' package [25]. For Cox modeling of COVID-19 infection events with vaccination or serological test results as independent variables, the respective time-dependent covariates were constructed as suggested by Therneau et al. using the 'tmerge' function of the 'survival' $\mathrm{R} /$ Bioconductor package [23]. We generated plots in $\mathrm{R}$ using the 'ggplot2' [26] and 'NMF' [27] packages.

\section{Results}

One hundred and seventy-five patients treated with dialysis (152 hemodialysis and 23 peritoneal dialysis), 252 kidney transplant patients and 71 nephrology healthcare team control participants provided specimens for serological analyses. Table 1 summarizes their demographic and clinical characteristics. Specimens were given before, between and/or after administration of vaccine doses and COVID-19 infections, as described in Table $\mathrm{S} 1$ (online supplement). Infections occurred prior to vaccine availability in $7.0 \%$, $9.1 \%$ and $9.1 \%$ of control, dialysis-treated and transplant participants, respectively $(\mathrm{p}=\mathrm{NS})$. The cumulative PCRverified case rate in Jerusalem by the same cutoff date was 
Table 1 Demographic and clinical characteristics according to study group

\begin{tabular}{|c|c|c|c|c|}
\hline & Control, $\mathrm{N}=71$ & Dialysis, $N=175$ & Transplant, $\mathrm{N}=252$ & p-value \\
\hline \multicolumn{5}{|l|}{ Age, years } \\
\hline Mean (SD) & $43.6(14.3)$ & $65.1(15.0)$ & $53.5(14.4)$ & $<0.001$ \\
\hline \multicolumn{5}{|l|}{ Sex } \\
\hline Female & $46(64.8)$ & $70(40.0)$ & $84(33.3)$ & \multirow[t]{2}{*}{$<0.001$} \\
\hline Male & $25(35.2)$ & $105(60.0)$ & $168(66.7)$ & \\
\hline \multicolumn{5}{|l|}{ Hypertension } \\
\hline No & $69(97.2)$ & $102(58.3)$ & $170(67.5)$ & \multirow[t]{2}{*}{$<0.001$} \\
\hline Yes & $2(2.8)$ & $73(41.7)$ & $82(32.5)$ & \\
\hline \multicolumn{5}{|l|}{ Diabetes } \\
\hline No & 70 (98.6) & $118(67.4)$ & $203(80.6)$ & \multirow[t]{2}{*}{$<0.001$} \\
\hline Yes & $1(1.4)$ & $57(32.6)$ & $49(19.4)$ & \\
\hline \multicolumn{5}{|l|}{$\mathrm{CHF}$} \\
\hline No & $71(100)$ & $148(84.6)$ & $248(98.4)$ & \multirow[t]{2}{*}{$<0.001$} \\
\hline Yes & $0(0)$ & $27(15.4)$ & $4(1.6)$ & \\
\hline \multicolumn{5}{|l|}{ COPD } \\
\hline No & $71(100)$ & 169 (96.6) & $247(98.0)$ & \multirow[t]{2}{*}{0.238} \\
\hline Yes & $0(0)$ & $6(3.4)$ & $5(2.0)$ & \\
\hline \multicolumn{5}{|l|}{ Cancer } \\
\hline No & 70 (98.6) & $164(93.7)$ & $240(95.2)$ & \multirow[t]{2}{*}{0.269} \\
\hline Yes & $1(1.4)$ & $11(6.3)$ & $12(4.8)$ & \\
\hline \multicolumn{5}{|l|}{ Prior COVID-19 } \\
\hline No & $66(93.0)$ & $159(90.9)$ & $229(90.9)$ & \multirow[t]{2}{*}{0.848} \\
\hline Yes & $5(7.0)$ & $16(9.1)$ & $23(9.1)$ & \\
\hline \multicolumn{5}{|c|}{ Vaccine inoculations } \\
\hline 0 & $12(16.9)$ & $31(17.7)$ & $41(16.3)$ & \multirow[t]{3}{*}{0.732} \\
\hline 1 & $3(4.2)$ & $6(3.4)$ & $16(6.3)$ & \\
\hline 2 & $56(78.9)$ & 138 (78.9) & $195(77.4)$ & \\
\hline \multicolumn{5}{|c|}{ Time from Tx, years } \\
\hline Median (range) & & & $4.0(0.25-49.0)$ & \\
\hline \multicolumn{5}{|c|}{ Dialysis vintage, years } \\
\hline Median (range) & - & $2.8(0.47-18.6)$ & - & - \\
\hline \multicolumn{5}{|c|}{ Urea reduction rate, $\%$} \\
\hline Mean (SD) & - & $72.1(8.2)$ & - & - \\
\hline \multicolumn{5}{|l|}{ Creatinine, $\mu \mathrm{mol} / \mathrm{l}$} \\
\hline Median (IQR) & $67.5(16.5)$ & - & $123.3(73.4)$ & $<0.001$ \\
\hline \multicolumn{5}{|c|}{$\mathrm{eGFR}, \mathrm{ml} / \mathrm{min} / 1.73 \mathrm{~m}^{2}$} \\
\hline Mean (SD) & $92.4(17.5)$ & - & $52.8(21.8)$ & $<0.001$ \\
\hline \multicolumn{5}{|l|}{ Tacrolimus $^{\mathrm{a}}, \mathrm{ng} / \mathrm{ml}$} \\
\hline Mean (SD) & - & - & $6.8(1.9)$ & - \\
\hline \multicolumn{5}{|l|}{ Hemoglobin, g/dl } \\
\hline Mean (SD) & $13.6(1.6)$ & $10.5(1.1)$ & $12.8(1.8)$ & $<0.001$ \\
\hline WBC, count per $\mu$ & & & & \\
\hline Mean (SD) & $7.6(1.9)$ & $7.6(2.7)$ & $8.4(2.4)$ & 0.004 \\
\hline Lymphocytes, per & & & & \\
\hline Mean (SD) & $2.2(0.7)$ & $1.2(0.6)$ & $1.7(0.9)$ & $<0.001$ \\
\hline
\end{tabular}

${ }^{\text {a }}$ Tacrolimus in $83 \%$, everolimus $9 \%$, cyclosporine (multiplied by 0.06 ) $7 \%$, sirolimus $1 \%$ 


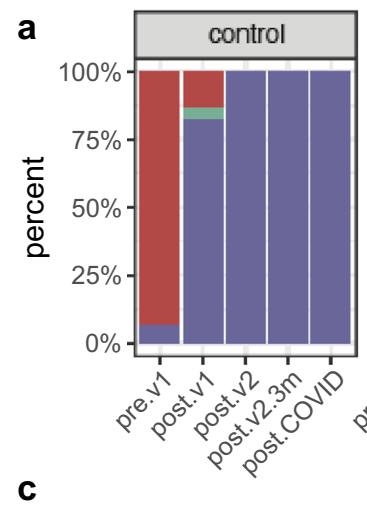

.

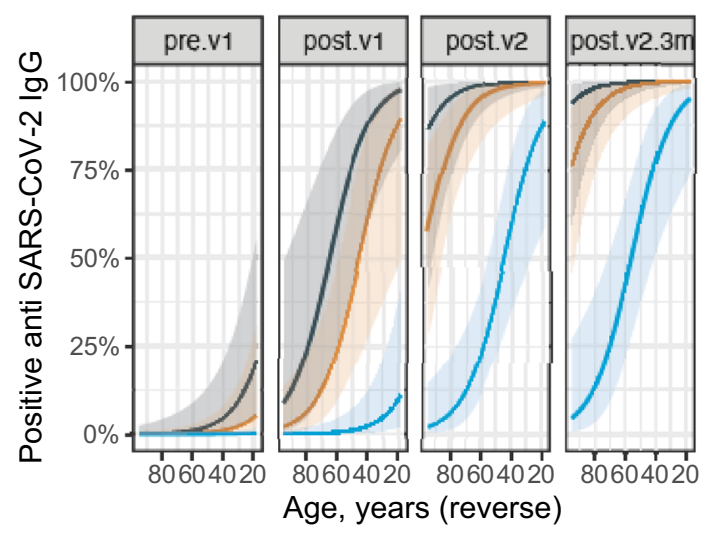

Fig. 1 Qualitative results of anti S1/S2 SARS-CoV-2 IgG serology testing. a Distribution of categorical results among the study groups along the study time points. Patients with positive or borderline serology at the baseline (pre.v1*) time point were assumed to have recovered from COVID-19 infection that was not otherwise suspected or diagnosed. b Model prediction of IgG positivity rates in the 3 study groups according to the timing of testing in relation to the 1st vaccination. The generalized linear mixed effects model also included a time $\times$ group interaction term, age and a patient identifier. c Model prediction of IgG positivity rates in the 3 study groups at different time points according to the participant's age. The generalized linear

similar to our control group, $6.75 \%[28,29]$. However, $20 \%$, $31 \%$ and $17 \%$ of our respective cases were identified solely via positive serology, without documentation of positive PCR tests.

Of our study participants, $82-83 \%$ (varying by group) received at least 1 dose of tozinameran and $77-79 \%$ received 2 inoculations, while among participants who were not infected with COVID-19 prior to vaccine availability, complete vaccination rates were $83-85 \%$. Figure S1 in the online supplement indicates the timeline of COVID-19 infections in our cohort (beginning at the epidemic onset) and of vaccination events.

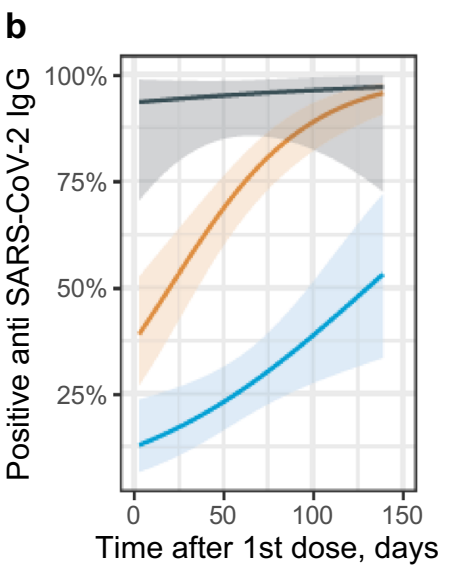

d

Factors associated with humoral non-response to vaccination

\begin{tabular}{|llccc|}
\hline Factor & Reference/units & $\begin{array}{c}\text { Odds } \\
\text { ratios }\end{array}$ & $\begin{array}{c}\text { Confidence } \\
\text { Intervals }\end{array}$ & p-value \\
\hline Dialysis group & control group & 39 & $6.0-2.6 \times 10^{2}$ & $<0.001$ \\
Transplant group & control group & $7.1 \times 10^{2}$ & $7.1-7.0 \times 10^{3}$ & $<0.00001$ \\
Time post 1st vaccine & days & 0.97 & $0.96-0.98$ & $<0.00001$ \\
Age >58 years (median) & $\leq 58$ years & 3.7 & $1.6-8.8$ & $<0.01$ \\
Cancer diagnosis & no cancer & 5.9 & $1.3-27$ & $<0.05$ \\
Diabetes & no diabetes & 2.6 & $1.1-6.4$ & $<0.05$ \\
Lymphocyte count & per 1,000 cellspll & 0.49 & $0.29-0.82$ & $<0.01$ \\
Vitamin D level & per 1 ng/ml & 0.94 & $0.89-1.00$ & $<0.05$ \\
\hline
\end{tabular}

mixed effects model also included a patient identifier. d Factors associated with humoral non-response to vaccination according to generalized linear mixed effects models with group, age, time from 1st vaccination and each one of the depicted additional variables separately. The dependent variable was categorical $(\geq 19 \mathrm{AU} / \mathrm{ml})$ antibody response after either 1 or 2 vaccine doses. Measurements from previously infected patients were not included. Definitions: pre.v1, before vaccination; post.v1, between vaccinations; post.v2, up to 10 weeks after the 2 nd vaccine; post.v $2.3 \mathrm{~m}$, more than 10 weeks after the 2 nd vaccine ( 3 months post 1 st vaccine); post.COVID, after COVID-19 infection (regardless of vaccination status)

\section{Serological results}

Figure 1a and Table S1 present the binary serological outcome among the 3 groups of participants at several occasions in relation to vaccination and infection. While $100 \%$ of control subjects generated positive antibody levels when sampled 22-71 days after the first vaccination (namely, 1-50 days after being fully vaccinated), only $70 \%$ of dialysis patients and $28 \%$ of transplant recipients achieved such levels when tested at the same time window, increasing to $79 \%$ and $42 \%$, respectively, 3 months or more after the first shot (range 90-139 days). In unvaccinated COVID19 survivors, the respective positive antibody rates were $100 \%$, 
a

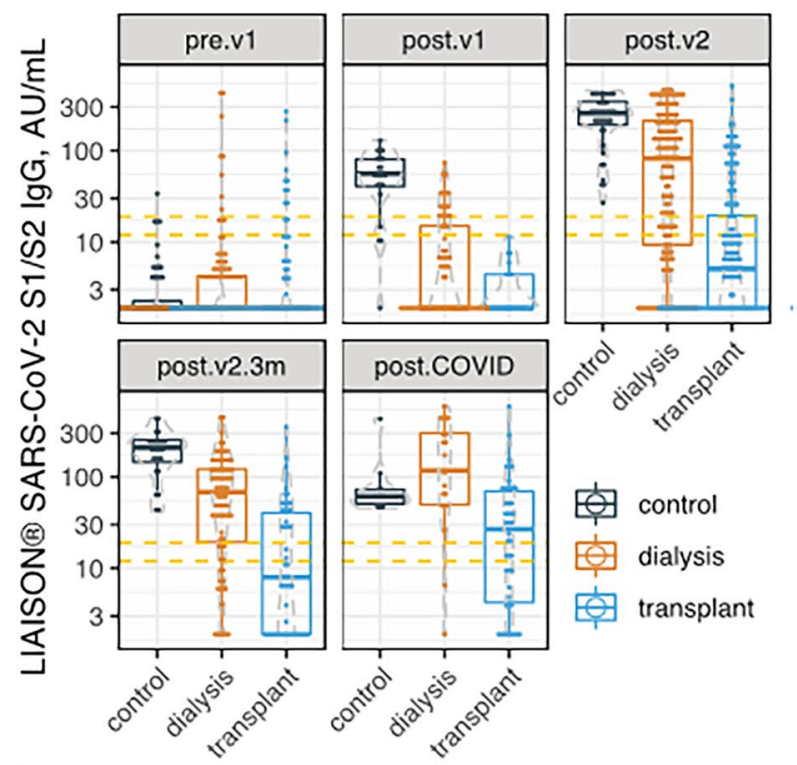

b

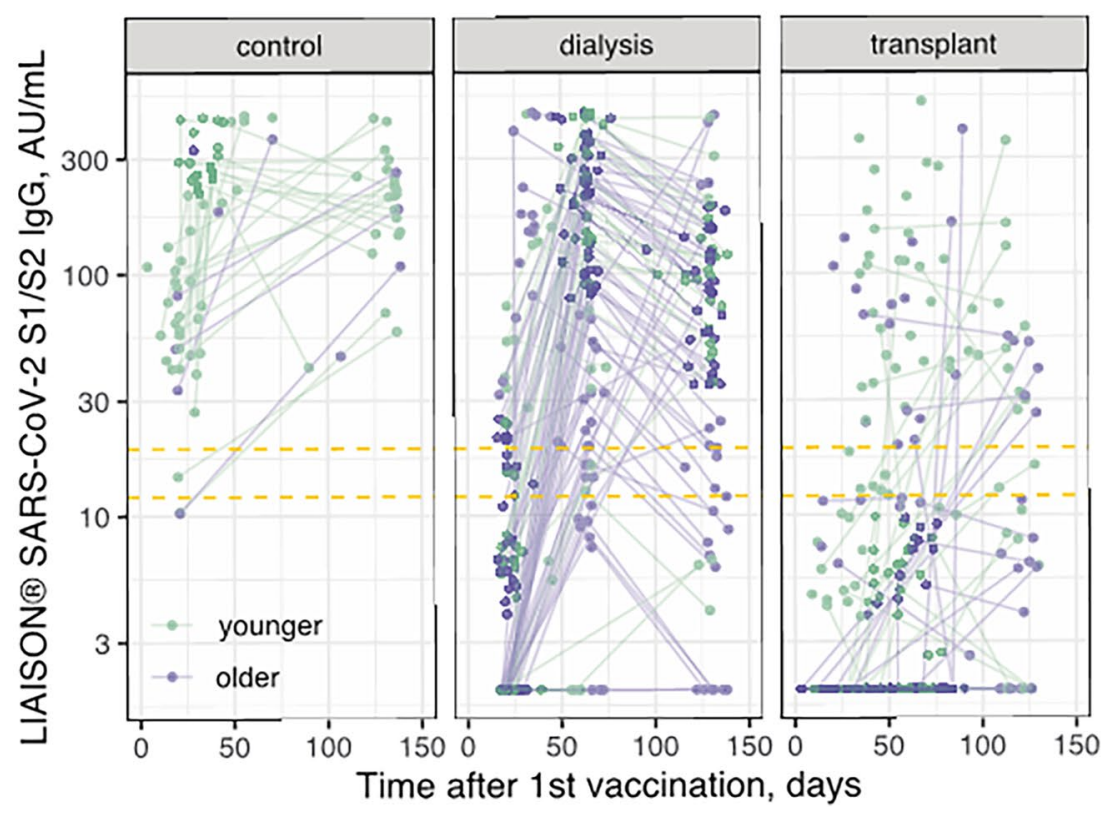

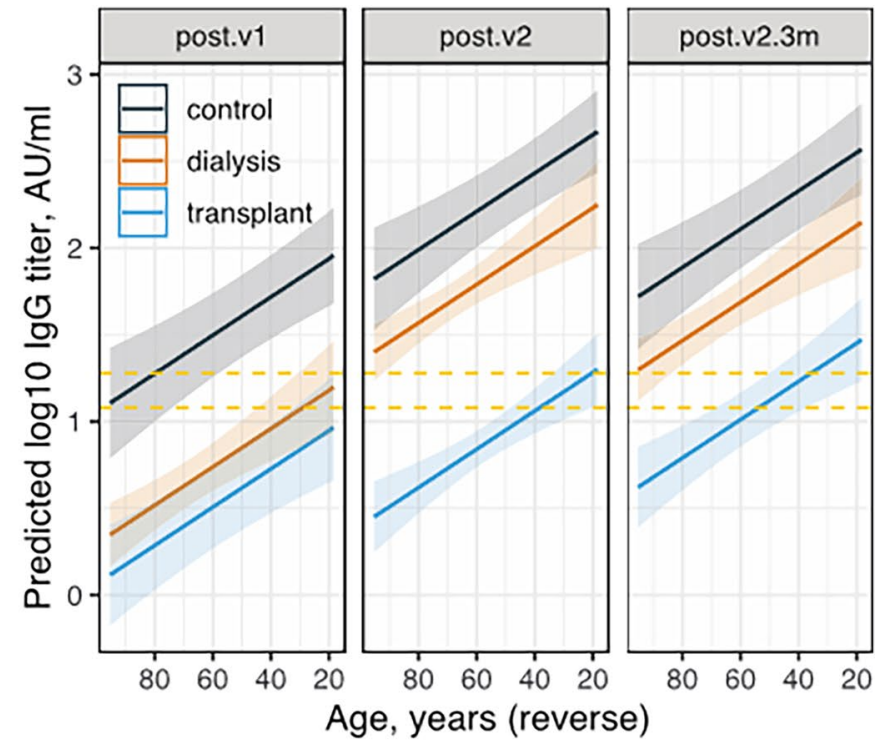

d

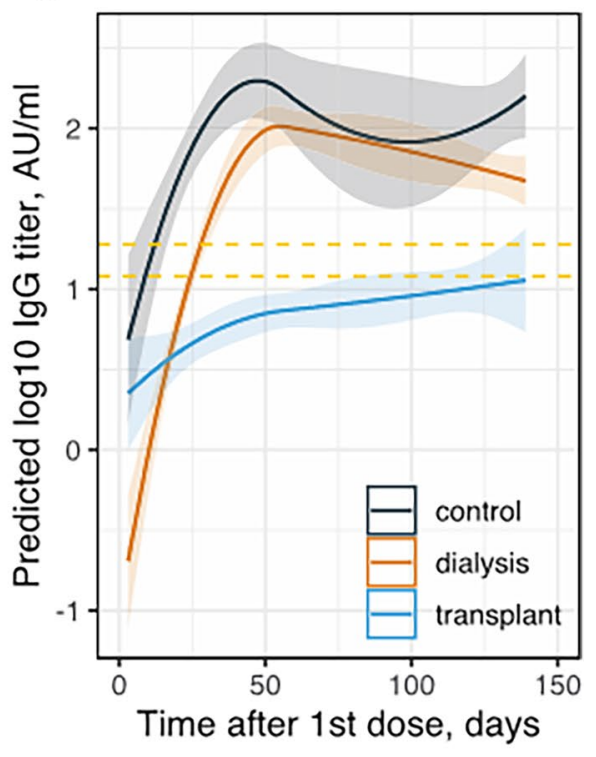

Fig. 2 Numerical results of anti S1/S2 SARS-CoV-2 IgG serology testing. a Dot- and box-plots showing antibody concentrations (log scale) in the 3 study groups at 5 specified time points. b Scatter plot showing antibody levels (log scale) versus time elapsed after 1st vaccine in the 3 study groups. Repeat measurements from the same participant are connected with lines. Measurements from previously infected patients were excluded. c Model prediction of IgG levels by age in the 3 study groups at different time points. The linear mixed

94\% and 69\%. Predictors of lack of anti-spike IgG response after single or double vaccine inoculation were: being a dialysis (odds ratio $=39,95 \%$ CI $\left.6.0-2.6 \times 10^{2}, \mathrm{p}<0.001\right)$ or transplant patient $\left(\mathrm{OR}=7.1 \times 10^{2}, 95 \% \mathrm{CI} 7.1-7.0 \times 10^{3}, \mathrm{p}<0.00001\right)$ compared to effects model included a group $\times$ time point interaction term and a patient identifier. d Model prediction of IgG levels versus time after 1 st vaccination in the 3 study groups. The linear mixed effects model included age, a group $\times$ time past first vaccination (as polynomial splines) interaction term and a patient identifier. See Fig. 1 legend for time point definition. The dashed yellow lines represent the equivocal concentration range

control, shorter time interval between immunization and testing $(\mathrm{OR}=0.97$ per day, $95 \%$ CI $0.96-0.98, \mathrm{p}<0.00001$, Fig. 1b), age above the median (58 years) $(\mathrm{OR}=3.7,95 \% \mathrm{CI} 1.6-8.8, \mathrm{p}<0.01$, and see Fig. 1c), cancer diagnosis $(\mathrm{OR}=5.9,95 \% \mathrm{CI} 1.3-27$, 
$\mathrm{p}<0.05)$ or diabetes $(\mathrm{OR}=2.6,95 \% \mathrm{CI} 1.1-6.4, \mathrm{p}<0.05)$, as well as lower lymphocyte count $(\mathrm{OR}=0.49,95 \%$ CI $0.29-0.82$, per 1,000 cells/ $\mu \mathrm{l}, \mathrm{p}<0.01$ ) and (in subjects with available data) lower vitamin $\mathrm{D}$ levels $(\mathrm{OR}=0.94,95 \% \mathrm{CI} 0.89-1.00$, per $1 \mathrm{ng} /$ $\mathrm{ml}, \mathrm{p}<0.05)$.

\section{Quantitative serological results}

Figure 2a and Table S2 display the numerical serological outcome among the 3 groups at several time points, in relation to vaccination and infection. The quantitative levels parallel the qualitative results presented above. Linear mixed effects models indicated that in addition to the time point of testing, $\operatorname{IgG}$ levels depended on the study group, being

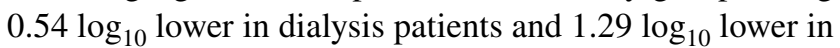
transplant patients, compared to controls (both $\mathrm{p}<0.00001$ ). $\log _{10}$ titers were 0.01 lower per year of age $(\mathrm{p}<0.00001)$. In addition, higher lymphocyte counts $\left(0.12 \log _{10}\right.$ per 1,000 cells/ $\mu \mathrm{l}, \mathrm{p}<0.01)$ and lower ferritin levels $\left(0.03 \log _{10}\right.$ per $100 \mathrm{ng} / \mathrm{ml}, \mathrm{p}<0.05$ ) were linked with higher antibody levels. Figure $2 \mathrm{~b}$ depicts antibody levels in participants that were fully vaccinated according to the time elapsed from the first dose. Predictions based on linear mixed effects models (with time post-vaccination introduced as an independent variable using splines and the time $\times$ group interaction also included in the model) are shown in Fig. 2c, d. A plateau is notable to emerge in controls and dialysis patients at $\sim 55$ days post 1st vaccination, while in transplant recipients a mild incline in antibody titers may persist beyond this period.

\section{Group-specific associations}

In transplant recipients, the step-up in antibody levels after the 2 nd vaccination was significantly greater in younger than the median ( $<55$ years) compared to older patients (Figure S2). In fact, 3 months or longer after the first dose, $60 \%$ of younger patients but only $24 \%$ of older transplant patients had a positive antibody response. Time from transplantation was associated with antibody titers in a non-linear manner; compared to patients transplanted 6-18 months before antibody testing (having lowest levels), recipients less than 6 months from transplantation date had $0.92 \log _{10}$ higher levels $(\mathrm{p}<0.01)$ and recipients of 18 months from transplantation or longer duration had $0.30 \log _{10}$ higher levels $(p<0.01)$. In models including age, timing of antibody testing and time after transplantation, antibody concentrations were $0.20 \log _{10}$ lower in patients with a diagnosis of hypertension $(\mathrm{p}<0.05)$ and $0.51 \log _{10}$ lower with a diagnosis of anemia $(\mathrm{p}<0.05)$. The aggregated immunosuppressive drug level-tacrolimus, cyclosporin (transformed by multiplying by 0.06), everolimus and sirolimus - was also linked with lower titers $\left(0.063 \log _{10}\right.$ per $n g / m l, p<0.05$, Figure $\mathrm{S} 2 \mathrm{c})$, however this association was not significant when accounting for time after transplantation $\left(0.055 \log _{10}\right.$ lower per $\mathrm{ng} / \mathrm{ml}, \mathrm{p}=0.08$ ). Serum creatinine and estimated glomerular filtration rate (CKD-EPI equation) did not associate with antibody levels.

In dialysis patients, unique predictors of antibody levels were: (1) modality; peritoneal dialysis patients had 0.41 higher $\log _{10}$ titer compared to hemodialysis patients (adjusted for age and time post-vaccination, $p<0.01$ ). (2) Ferritin levels were linked with $\operatorname{lower} \log _{10}$ titers $(0.03$ per $100 \mathrm{ng} / \mathrm{ml}, \mathrm{p}<0.05$ ) (Figure S3). Conversely, dialysis vintage, comorbidity conditions and averaged urea reduction rate (in hemodialysis patients) were not associated with antibody concentrations.

\section{Occurrence of COVID-19}

Ninety six study participants had COVID-19 infection before or after the vaccination period, up until 31-May2021 (Figure S1b). Cumulative rates were 15.5\% in controls, $20.6 \%$ in transplant patient and $18.3 \%$ in dialysis patients $(\mathrm{p}=\mathrm{NS})$. The respective cumulative PCR-verified case rate in Jerusalem was $13.58 \%$ [28, 29]. Vaccine inoculations, introduced as time-varying covariates, are seen in Fig. 3a (and Figure S4a) to inversely associate with COVID-19 infection risk, after vaccine availability. This relationship was marginally stronger in controls compared to both ESRD groups (p-values for the interactions $\sim 0.1$ ), but was significantly weaker in older (58.1-95 year old) compared to younger (18.2-57.9 year old) subjects (Figs. 3b, S4b), possibly owing to lower baseline risk in this subgroup ( $\mathrm{p}$-value for the interaction 0.013). Also, vaccine breakthrough infections in dialysis patients and particularly transplant recipients were of lesser severity compared to infections in unvaccinated patients (not shown).

Moreover, positive (including borderline) anti-SARSCoV-2 IgG serology after vaccination was linked with lower risk of COVID-19 infection (Figs. 3c, S4c), hazard ratios 0.23 (95\% CI 0.05-0.99). Importantly, not a single participant became infected with a most recent anti S1/S2 IgG antibody test result of $59 \mathrm{AU} / \mathrm{ml}$ or higher (Fig. 3d and Supplementary Text). Likewise, quantitative IgG titers were linked with significantly lower COVID-19 risk (Fig. 3e, S4d). See additional analyses including mortality as supplementary material (Supplementary Text and Figure S5).

\section{Post-COVID-19 serology}

SARS-CoV-2 S1/S2 IgG antibody concentrations after COVID-19 infection were lower compared to vaccine-driven antibodies in controls. However, in transplant recipients and dialysis patients the opposite was true (Fig. 4). 
a

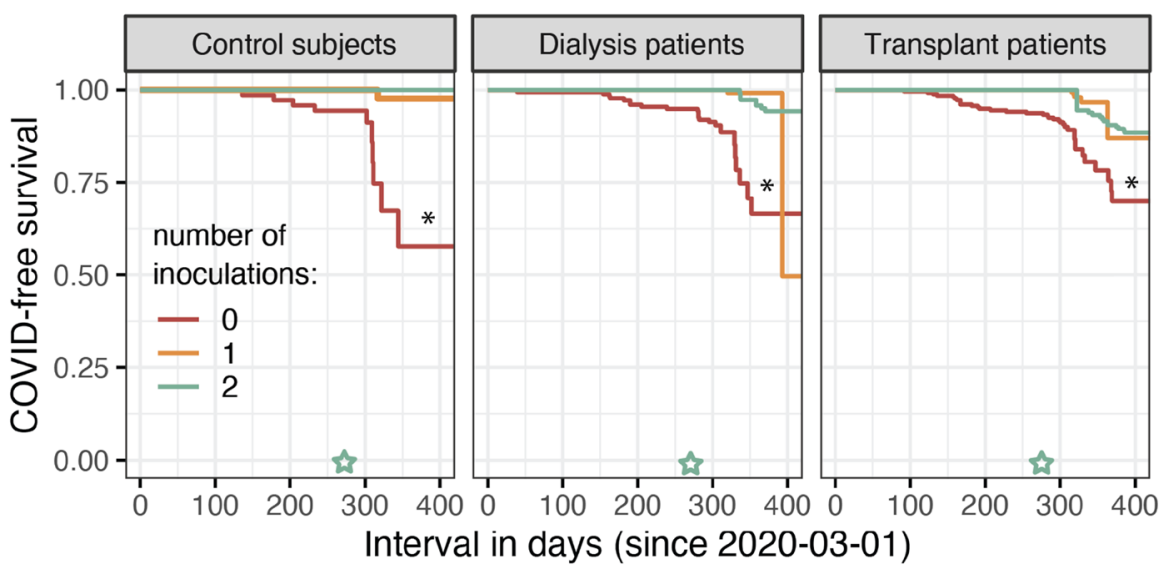

b

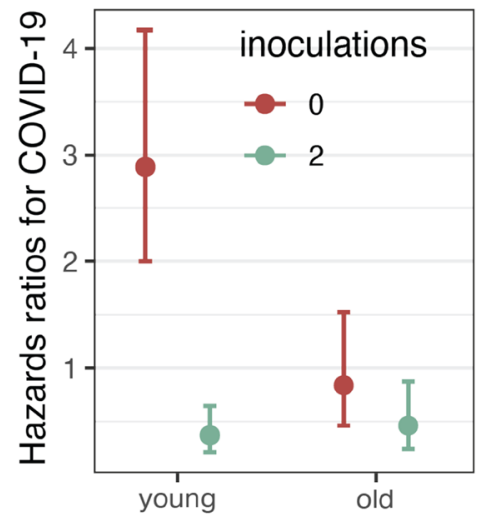

C


Fig. 3 Associations between vaccine inoculations, anti S1/S2 IgG test results and COVID-19 infection. a COVID-19 infection-free survival from epidemic onset according to vaccination status as a timevarying covariate (see "Methods"), split by study group. *, p $<0.05$ for 2 vs. 0-1 inoculations. b Risk of COVID-19 infection by inoculation status and age group ( $<58$ year old vs. $\geq 58$ year old). The model also included a patient identifier and was stratified by study group. c COVID-19 events presented as Kaplan Meier curves according to

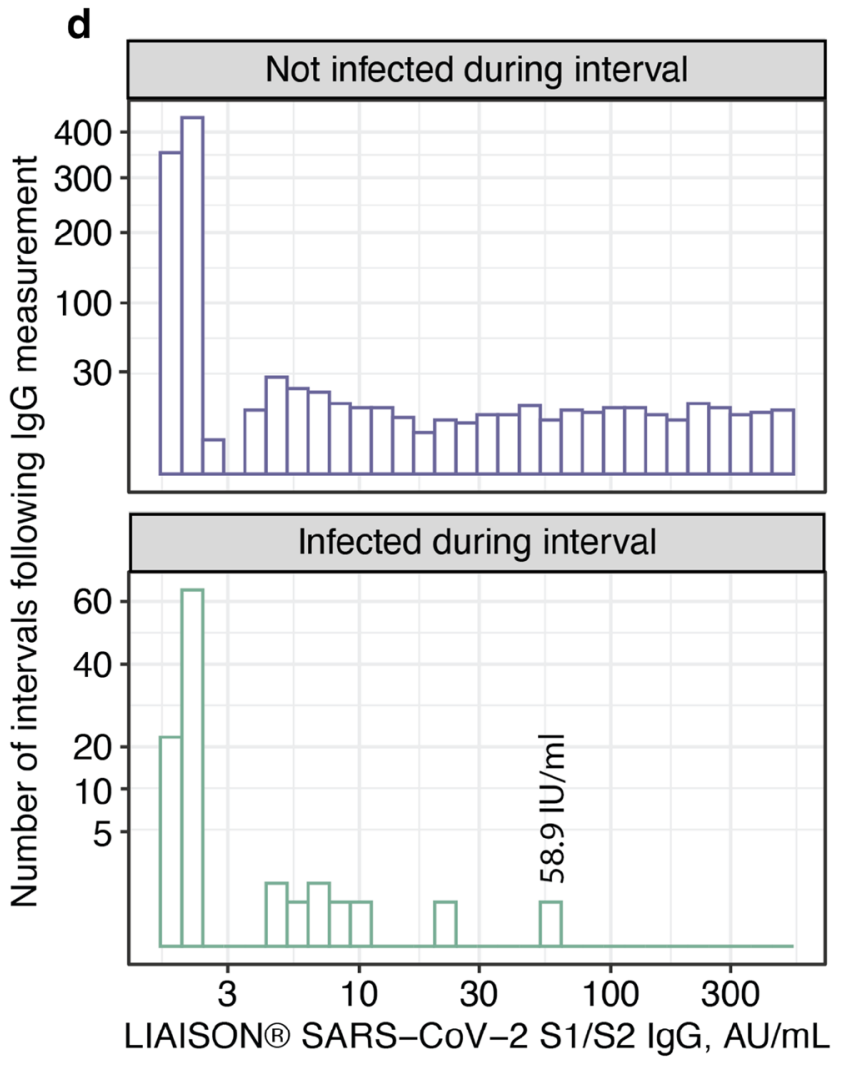

IgG serology status. d Histograms showing the distribution of serology results (logarithmic scale) according to whether or not the participant became infected after the test was performed, but prior to the subsequent test. A putative protective cutoff is observed at $\sim 60 \mathrm{AU} /$ $\mathrm{ml}$. e Determinants of COVID-19 risk in a model including $\log \mathrm{IgG}$ level as a time-varying covariate. The older vs. younger break is the median age, 58 years. In a and $\mathbf{c}$ the green star marks the beginning of the vaccination period 
a


b

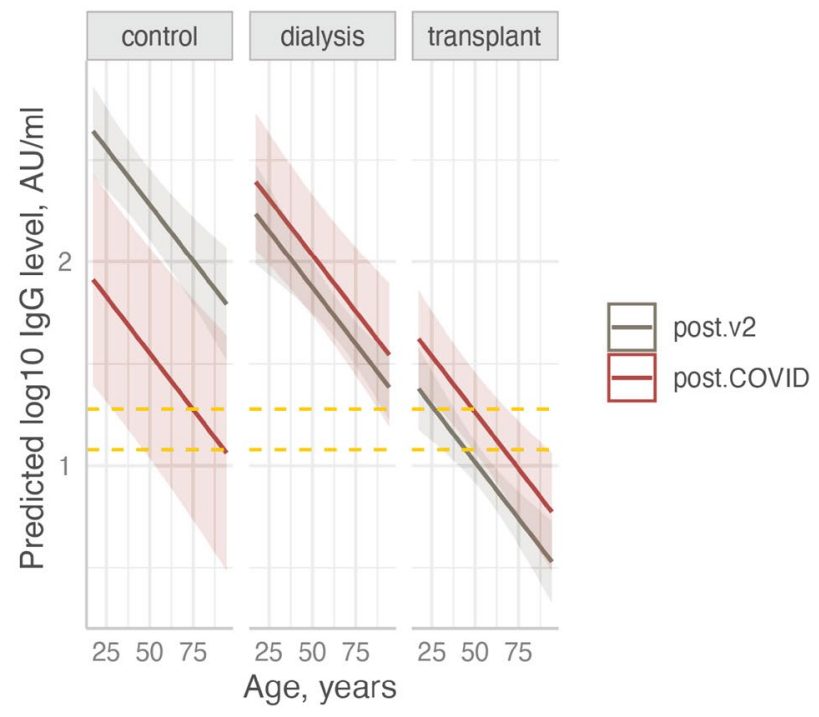

Fig. 4 Comparison of post-vaccination with post-COVID-19 infection anti S1/S2 SARS-CoV-2 IgG levels. a Dot- and violin plots showing IgG concentrations post-COVID-19 vaccination (post.v2) or infection (post.COVID). Also shown are the means and confidence limits (based on the t-distribution). b Model prediction of IgG concentration versus age, post-infection and post-vaccination, in the three study groups, showing higher predicted levels post-vaccine in controls but not in the ESRD groups. The linear mixed effect model also included the time post-vaccination (modeled with polynomial splines) and a patient identifier. Dashed yellow lines cover the equivocal antibody level range.

\section{Discussion}

This prospective study was designed to follow serum antibody levels after COVID-19 vaccination and/or disease in patients requiring chronic renal replacement therapy, and to estimate associations of vaccine inoculation and the resulting humoral response with subsequent COVID-19 infections. Healthcare team personnel (nursing, medical, assisting and administrative staff, working in the respective dialysis units and/or in the transplantation clinic) served as controls. Anti-spike antibody positivity rates and levels did not differ between groups at baseline. However, dialysis and more prominently kidney transplant patients had significantly lower positive response rates and antibody levels after both the first and second vaccine administration compared to the healthcare controls. These findings are in line with previous studies in kidney dialysis and transplant patients [30-32]. Not previously reported, we found that immunization using the tozinameran mRNA vaccine was linked with lower risk of COVID-19 infection in all groups. This effect was smaller in patients compared to controls. Also, we propose a putative protective S1/S2 antibody level cutoff of 60 $\mathrm{AU} / \mathrm{ml}$ (DiaSorin), consistent with a prior publication [33].

Prior to vaccination, positive serology was found in $<10 \%$ of participants, indicating the prevalence of past infection with SARS-CoV-2 virus. About one third of these infections were not detected or suspected previously, despite screening measures in dialysis patients - questioning for possible contacts with verified COVID-19 patients, risk factors for exposure (as recent flight, attending crowded events or living in communities with high rates of infection), and symptoms that can suggest active infection, as well as measuring body temperature and repeated SARS-CoV-2 nucleic acid quantification by nasal swabs after exposure.

Only $28 \%$ of transplant patients and $70 \%$ of dialysis patients had a positive (not including borderline) antibody response after the second shot of the vaccine, improving by 3 months to $42 \%$ and $79 \%$, respectively, yet still having significantly lower antibody titers compared with controls. This raises the question whether a vaccine boost or higher doses are needed in this patient population, as was previously reported for the hepatitis B vaccine [13].

Strikingly, many fully vaccinated transplant patients were identified with no detectable antibodies (and some developed severe COVID-19 after infection). Similar findings, of reduced or lack of humoral response to the two-dose regimen of tozinameran, were reported previously in dialysis patients [31, 34-36], kidney transplant [30, 37, 38], and other solidorgan recipients [39-41], although follow-up periods were usually much shorter than in our study and there were typically no investigations or links with subsequent infection.

During the 5.5 month study period, infection rates in Israel, Jerusalem included, dropped drastically. Despite these circumstances, we found that COVID-19 infection following vaccination was independently associated with lower post-vaccination antibody titers, implying benefit from immunization with the mRNA vaccine on top of herd immunity. This association, suggesting a vaccine-related beneficial effect, was reported previously with neutralizing antibody assays [42] but not with antibodies detected by 
common immunoassays. This is of clinical and epidemiological importance, because by identifying individuals at high risk of infection with readily available tests, one can apply individualized protective measures, while easing on the general population, or introduce additional vaccination boosts for specific vulnerable groups.

The findings that lower lymphocyte counts and higher serum levels of immunosuppressive medications are associated with lesser antibody response also suggest the need for additional vaccination for immunosuppressed patients. Intriguingly, controls mounted lower anti S1/S2 concentrations after infection compared to vaccination, while the reverse was true in patients. A partial explanation for the latter finding in transplant recipients is the practice of lowering immunosuppressive medications (aside from corticosteroids) during and shortly after infection.

There are several limitations to our study. This is a single-center study, and may not represent the larger ESRD community when considering future immunization policies, though we believe that combined with contemporary publications the study is large enough to facilitate further research. A longer follow-up period can enrich the data on antibody titers, and the recurrent escalation of COVID-19 incidence in Israel means that new data regarding protection will be available [43]. There are significant age differences between the control group and the dialysis patient group, which is compatible with the average age of dialysis patients. As significant age-related differences in antibody concentrations were found in the vaccine's clinical trials, where older individuals had lower antibody concentrations (also observed among our study participants), we adjusted for age in the statistical models, and are thus reporting a significant age-independent relationship between study group and antibodies. Additionally, COVID-19 infection was defined as a positive PCR, regardless of symptoms, but routine nucleic acid screening was not performed, and therefore we might have missed asymptomatic patients. Lastly, we did not report in this study ongoing investigation of cellular immunity or non-IgG antibodies which could add to our understanding of the immune response and protection after vaccination.

In conclusion, we show that kidney transplant and dialysis patients exhibit impaired humoral response to two doses of tozinameran, a prototype of mRNA vaccination, manifested either by negative immunoassay or lower concentrations compared to healthy controls. Of note, a small rise in antibody levels and proportion of responding patients is evident beyond three months, suggesting a different time scale of the immune response. Additionally, we show an inverse association of $\mathrm{IgG}$ concentrations with the risk of contracting COVID-19 after immunization, and propose $60 \mathrm{AU} / \mathrm{ml}$ as a putative protective cutoff (using the LIAISON SARSCoV-2 S1/S2 IgG serology test by DiaSorin). We suggest testing immune-compromised patients for COVID-19 IgG antibodies in order to identify high-risk patients, and to expand research regarding the effects of booster dosing.

Supplementary Information The online version contains supplementary material available at https://doi.org/10.1007/s40620-021-01210-y.

Author contributions Study conception and design-IZB-D, AK, MDE, DGW. Data acquisition including patient recruitment-KT, TA, RB, RA, HA, MA, IB, HP-C, AI, RA, IM-YL, EOD, AB. Data analysis-IZB-D. Data interpretation-IZB-D, YO, MDE. Drafting the manuscript-YO, IZB-D, MDE. Revising the manuscript-KT, AK.

Funding There was no external funding for this study. The Ministry of Health of the State of Israel supported this study by supplying serology kits to the hospital's virology laboratory.

\section{Declarations}

Conflict of interest On behalf of all authors, the corresponding author states that there is no conflict of interest.

\section{References}

1. Plotkin S, Plotkin S (2011) The development of vaccines: how the past led to the future. Nat Rev Microbiol. https://doi.org/10.1038/ nrmicro2668

2. Plotkin S, Plotkin S (2008) A short history of vaccination. In: Plotkin S, Orenstein W, Offit P (eds) Vaccines. Amsterdam, ElsevierSaunders, pp 1-16

3. Haas E, Angulo F, McLaughlin J et al (2021) Impact and effectiveness of mRNA BNT162b2 vaccine against SARS-CoV-2 infections and COVID-19 cases, hospitalisations, and deaths following a nationwide vaccination campaign in Israel: an observational study using national surveillance data. Lancet (London, England). 397:10287. https://doi.org/10.1016/S0140-6736(21)00947-8

4. McDonald I, Murray S, Reynolds C, Altmann D, Boyton R (2021) Comparative systematic review and meta-analysis of reactogenicity, immunogenicity and efficacy of vaccines against SARS-CoV-2. NPJ Vaccines. https://doi.org/10.1038/ s41541-021-00336-1

5. Hilbrands L, Duivenvoorden R, Vart P et al (2020) COVID-19-related mortality in kidney transplant and dialysis patients: results of the ERACODA collaboration. Nephrol Dial Transplant. https:// doi.org/10.1093/ndt/gfaa261

6. Meo S, Bukhari I, Akram J, Meo A, Klonoff D (2021) COVID19 vaccines: comparison of biological, pharmacological characteristics and adverse effects of Pfizer/BioNTech and Moderna Vaccines. Eur Rev Med Pharmacol Sci. https://doi.org/10.26355/ eurrev_202102_24877

7. Verna E, Landis C, Brown R et al (2021) Factors Associated with Readmission in the US Following Hospitalization with COVID19. Clin Infect Dis. https://doi.org/10.1093/cid/ciab464

8. Lano G, Braconnier A, Bataille S et al (2020) Risk factors for severity of COVID-19 in chronic dialysis patients from a multicentre French cohort. Clin Kidney J. https://doi.org/10.1093/ckj/ sfaa199

9. Johnson DW, Fleming SJ (1992) The use of vaccines in renal failure. Clin Pharmacokinetics. https://doi.org/10.2165/00003088199222060-00003 
10. Mastalerz-Migas A, Gwiazda E, Brydak LB (2013) Effectiveness of influenza vaccine in patients on hemodialysis-a review. Med Sci Monit. https://doi.org/10.12659/MSM.889671

11. Rautenberg P, Teifke I, Schlegelberger T, Ullmann U (1988) Influenza subtype-specific IgA, IgM and IgG responses in patients on hemodialysis after influenza vaccination. Infection. https://doi.org/ 10.1007/BF01644539

12. Beyer WE, Versluis DJ, Kramer P, Diderich PP, Weimar W, Masurel N (1987) Trivalent influenza vaccine in patients on haemodialysis: impaired seroresponse with differences for A-H3N2 and A-H1N1 vaccine components. Vaccine. https://doi.org/10.1016/ 0264-410x(87)90008-9

13. Yao T, Shao Z, Wu L et al (2021) Long-term persistent immunogenicity after successful standard and triple-dosed hepatitis B vaccine in hemodialysis patients: A 3-year follow-up study in China. Vaccine. https://doi.org/10.1016/j.vaccine.2021.03.074

14. Miskulin D, Weiner D, Manley H (2020) High-dose versus standard-dose influenza vaccine in hemodialysis patients. Am J Kidney Dis. https://doi.org/10.1053/j.ajkd.2019.09.019

15. Kim JU, Kim M, Kim S et al (2017) Dendritic cell dysfunction in patients with end-stage renal disease. Immune Netw. https://doi. org/10.4110/in.2017.17.3.152

16. Earle K, Ambrosino D, Fiore-Gartland A et al (2021) Evidence for antibody as a protective correlate for COVID-19 vaccines. Vaccine. https://doi.org/10.1016/j.vaccine.2021.05.063

17. Heldman M, Limaye A (2021) SARS-CoV-2 vaccines in kidney transplant recipients: will they be safe and effective and how will we know? J Am Soc Nephrol JASN. https://doi.org/10.1681/ASN. 2021010023

18. Kliger A, Silberzweig J (2021) COVID-19 and dialysis patients: unsolved problems in early 2021. J Am Soc Nephrol JASN. https://doi.org/10.1681/ASN.2020121766

19. Benenson S, Oster Y, Cohen M, Nir-Paz R (2021) BNT162b2 mRNA Covid-19 vaccine effectiveness among health care workers. N Engl J Med. https://doi.org/10.1056/NEJMc2101951

20. COVID-19-Government Data. https://data.gov.il/dataset/ covid-19

21. R: A Language and Environment for Statistical Computing. $R$ Foundation for Statistical Computing; 2021. https://www.R-proje ct.org

22. Bates D, Mächler M, Bolker B, Walker S. Fitting Linear MixedEffects Models Using lme4. Articles. J Stat Software. 2015;67 https://www.jstatsoft.org/index.php/jss/article/view/v067i01

23. Therneau TM, Grambsch PM (2000) Modeling survival data: extending the Cox model. Springer, New York

24. Lüdecke D (2021) sjPlot: Data Visualization for Statistics in Social Science. https://CRAN.R-project.org/package $=$ sjPlot

25. Lüdecke D (2021) ggeffects: tidy data frames of marginal effects from regression models. J Open Source Softw 3(26):772. https:// doi.org/10.21105/joss.00772

26. Wickham H (2016) ggplot2: elegant graphics for data analysis. Springer, New York

27. Gaujoux R, Seoighe C (2010) A flexible R package for nonnegative matrix factorization. BMC Bioinform. https://doi.org/10. 1186/1471-2105-11-367

28. COVID-19 City Table. State of Israel Ministry of Health. https:// data.gov.i1/dataset/covid-19/resource/8a21d39d-91e3-40db-aca1f73f7ab1df69. Accessed 24 Sep 2021

29. Population in Cities, 2021. Central Bureau of Statistics. https:// www.cbs.gov.i1/he/publications/LochutTlushim/2020/\%D7\%90\%
D7\%95\%D7\%9B\%D7\%9C\%D7\%95\%D7\%A1\%D7\%99\%D7\%99\% D7\%942020.xlsx. Accessed 24 Sep 2021

30. Rozen-Zvi B, Yahav D, Agur T et al (2021) Antibody response to SARS-CoV-2 mRNA vaccine among kidney transplant recipients: a prospective cohort study. Clin Microbiol Infect. https://doi.org/ 10.1016/j.cmi.2021.04.028

31. Grupper A, Sharon N, Finn T et al (2021) Humoral response to the Pfizer BNT162b2 vaccine in patients undergoing maintenance hemodialysis. Clin J Am Soc Nephrol: CJASN. https://doi.org/10. 2215/CJN.03500321

32. Grupper A, Rabinowich L, Schwartz D et al (2021) Reduced humoral response to mRNA SARS-CoV-2 BNT162b2 vaccine in kidney transplant recipients without prior exposure to the virus. Am J Transplant. https://doi.org/10.1111/ajt.16615

33. Rubio-Acero R, Castelletti N, Fingerle V et al (2021) In search of the SARS-CoV-2 protection correlate: head-to-head comparison of two quantitative S1 assays in pre-characterized oligo-/asymptomatic patients. Infect Dis Therapy. https://doi.org/10.1007/ s40121-021-00475-x

34. Anand S, Montez-Rath M, Han J et al (2021) Serial SARS-CoV-2 receptor-binding domain antibody responses in patients receiving dialysis. Ann Internal Med. https://doi.org/10.7326/M21-0256

35. Simon B, Rubey H, Treipl A et al (2021) Haemodialysis patients show a highly diminished antibody response after COVID-19 mRNA vaccination compared to healthy controls. Nephrol Dial Transplant. https://doi.org/10.1093/ndt/gfab179

36. Agur T, Ben-Dor N, Goldman S et al (2021) Antibody response to mRNA SARS-CoV-2 vaccine among dialysis patients-a prospectivecohort study. Nephrol Dial Transplant. https://doi.org/10. 1093/ndt/gfab155

37. Husain S, Tsapepas D, Paget K et al (2021) Post-vaccine antiSARS-CoV-2 spike protein antibody development in kidney transplants recipients. Kidney Int Rep. https://doi.org/10.1016/j.ekir. 2021.04.017

38. Korth J, Jahn M, Dorsch O et al (2021) Impaired humoral response in renal transplant recipients to SARS-CoV-2 vaccination with BNT162b2 (Pfizer-BioNTech). Viruses. https://doi.org/10.3390/ v13050756

39. Boyarsky B, Werbel W, Avery R et al (2021) Antibody Response to 2-Dose SARS-CoV-2 mRNA vaccine series in solid organ transplant recipients. JAMA. https://doi.org/10.1001/jama.2021. 7489

40. Wadei H, Gonwa T, Leoni J, Shah S, Aslam N, Speicher L (2021) COVID-19 infection in solid organ transplant recipients after SARS-CoV-2 vaccination. Am J Transplant. https://doi.org/10. 1111/ajt.16618

41. Peled Y, Ram E, Lavee J et al (2021) BNT162b2 vaccination in heart transplant recipients: clinical experience and antibody response. J Heart Lung Transplant. https://doi.org/10.1016/j. healun.2021.04.003

42. Sui Y, Bekele Y, Berzofsky J (2021) Potential SARS-CoV-2 immune correlates of protection in infection and vaccine immunization. Pathogens (Basel, Switzerland). https://doi.org/10.3390/ pathogens10020138

43. Bar-On Y, Goldberg Y, Mandel M et al (2021) Protection of BNT162b2 vaccine booster against Covid-19 in Israel. N Engl J Med. https://doi.org/10.1056/NEJMoa2114255

Publisher's Note Springer Nature remains neutral with regard to jurisdictional claims in published maps and institutional affiliations. 


\section{Authors and Affiliations}

Iddo Z. Ben-Dov ${ }^{1}$ (I) Yonatan Oster ${ }^{2} \cdot$ Keren Tzukert $^{1} \cdot$ Talia Alster $^{1} \cdot$ Raneem Bader $^{3} \cdot$ Ruth Israeli $^{1} \cdot$ Haya Asayag $^{1}$. Michal Aharon ${ }^{1} \cdot$ Ido Burstein $^{1} \cdot$ Hadas Pri-Chen $^{1}$ - Ashraf Imam ${ }^{3}$ - Roy Abel ${ }^{1}$ - Irit Mor-Yosef Levi ${ }^{1} \cdot$ Abed Khalaileh $^{3}$. Esther Oiknine-Djian ${ }^{2} \cdot$ Aharon Bloch $^{1} \cdot$ Dana G. Wolf ${ }^{2} \cdot$ Michal Dranitzki Elhalel $^{1}$

Iddo Z. Ben-Dov

iddo@hadassah.org.il

1 Department of Nephrology and Hypertension, Hadassah Medical Center, Faculty of Medicine, Hebrew University of Jerusalem, Kiryat Hadassah 1, 921120 Jerusalem, Israel
2 Department of Clinical Microbiology and Infectious Diseases, Hadassah Medical Center, Faculty of Medicine, Hebrew University of Jerusalem, Jerusalem, Israel

3 Department of Surgery, Hadassah Medical Center, Faculty of Medicine, Hebrew University of Jerusalem, Jerusalem, Israel 